\title{
Application of Smartphone Qrcode Scanner as a means of Authenticating Student Identity Card
}

\author{
Adeniyi Abidemi Emmanuel ${ }^{1}$, Amusan Elizabeth Adedoyin ${ }^{2}$, Olagunju Mukaila ${ }^{3}$ and Ogundokun Oluwaseun Roseline $^{1}$ \\ ${ }^{1}$ Department of Computer Science, College of Pure and Applied Sciences, Landmark University. \\ ${ }^{2}$ Department of Computer Science and Engineering, Faculty of Engineering and Technology, \\ Ladoke Akintola University of Technology, Ogbomoso. \\ ${ }^{3}$ Department of Computer Science, Faculty of Sciences, Federal University, Oye-Ekiti, Ekiti State.
}

ORCID: 0000-0002-2728-0116 (Adeniyi)

\begin{abstract}
The smartphone scanner is the best tool to authenticate any object with embedded Qr codes anytime and anywhere. This smartphone scanner will make it easier and simpler to encode the generated code without any difficulty. The need for this research is to enhance the quality time of authentication which can now be done anywhere and anytime without the problem of location and connectivity issue. However, smart card technology has not been fully embraced, particularly in developing countries like Nigeria because of our lack of innovation in information technology. As a solution, this work presents a real time student identity card authentication system with the aid of android phone camera scanner in built function. The developed system accepts as input, a matriculation number which is unique for every student and encrypts it into a two-dimensional bar code using a QR generator. The generated $\mathrm{QR}$ code is then embedded in the identity card alongside other details of the cardholder which produce a dynamic authentication output. A software application that functions as a QR scanner is pre-installed on the smartphone. This makes it possible to reverse this process during authentication for a reconciliation of the decrypted code with the student matriculation number. The result of the experiment reveals that the smartphone scanner is effective and faster to authenticate the embedded qr code trademark than any other means of authentication.
\end{abstract}

Keywords: Smartphone Camera, Identity Card, QR code, QR generator, QR scanner, Smartphone.

\section{INTRODUCTION}

Authentication of any object with trademark is indispensable in this era of Information Technology as production of fake items has become rampant. The use of smartphone scanner to verify the originality an item is necessary as it is fast and easy to implement and use (Bajpai and Agrawal, 2013). Authentication of a student identity card with the aid of a smartphone scanner is a method of identifying the quick response code trademark embedded in each student identity card. To automatically identify a student is a token of an eye, smartphone are increasingly being used. Authentication is the process of confirming the real identity of an attribute of a piece of data claimed true by an entity. A student Identity card is said to be the summary of a student's information and with the speedy increase of students in our institutions, it is now a must for every institution to have a means of automatic authentication of each student. It is highly essential for an educational institution to provide Identity card dynamically with quick response code embedded and that can easily be authenticated using a smartphone for every student.

Automatic Authentication of Student Identity card with Quick response code and smartphone system is actually means of maximize the usage of student ID card and also by doing that, it will upgrade the system that been used in the Identification of student card nowadays. With the successful implementation of dynamic Qr code in student identity cards, it can also help in other students activities within the campus, for example, student lecture attendance, library usage, hostel insurance and while taking the final examination. Other than that much higher institution still uses their own in a house security guard to check student in and out during peak hour. Rather than use a person, why not all higher institutions have their own automatic barrier that needs the student to swipe their own identity card before entering campus areas. This will totally increase the safety of their learning places.

The rapid development of various aspects of computer technology gives rise to the usage of various information technology gadgets in our day to day activities (Richard, 2102). In this research work, a quick response code is embedded to student identity card and smartphone is used to authenticate the encrypted embedded quick response code automatically which will redirect to a site to authenticate the encrypted code. This proposed work uses a web application to generate an embedded student identity card locally on the computer system and whenever the need arises for authentication of the card, the identity card is placed under smartphone camera, the smartphone scan the embedded quick response code and decrypt the encrypted details for verification. This process is purely automatic authentication process. It does not require manual entry on matriculation before performing the authentication process. 


\section{RELATED WORK}

There are many proposals for a student identity card with quick response code and smartphone. Most of them to focus on the application of static nature of qrcode verification which does not redirect to the webpage where the code is being generated and also internet connectivity of either the smartphone or the software application, while the closely related make use of manual input of matriculation number before authentication. In this section, we will mention briefly a few of these proposals.

Bajpai and Agrawal (2013) presented a paper on personalized data, signature and picture of cardholders were captured, their systems generated barcode in 2-dimensional formats which represents the captured data and embedded this barcode image on the identity card. The validation and authentication of this card they produced were done on an online centralized database. The intention of their authentication was to match the encrypted unique identity of the person in the barcode's image with the one in the online central database. The major shortcoming of their work was that solely depends on internet connectivity for its operation.

Singhal and pavithr (2015) use quick response code and smartphone for verification and authentication of an educational certificate. In their work, the QR code was embedded on the student certificate that decrypts the signature from the university's public key in order to generate the hash value for verification. For the certificate to pass the authentication process, the hashed value generated from the certificate must match that of the university's public key otherwise, the certificate will not pass the authentication process. This work was a purely online system which will render the authentication process unless whenever there is no internet connectivity.

Cho and Bae (2014); Masalha and Hirzallah (2014); Kumar and Kareemulla (2017) these researchers proposed an online attendance system for both the staff and student using QR code and Smartphone. In this work, the Staff is required to generate a QR code that would be scanned by the students and sent through the wireless network for necessary automatic attendance checking. In other to avoid proxy of attendance, Kumar and Kareemulla introduced fingerprint and voice verification to authenticate the attendance system.

Ayeleso, et al. (2017) proposed an embedded QR code on identity card to be given to students and introducing Smartphone App to authenticate the embedded QR code. Their proposed system is an offline system which does not require a network connection for authentication. The major shortcoming of their work is that student matriculation number has to be manually inputted before authentication.

The first three work reviewed earlier shows that their proposed systems work with the internet connection. That is their operation is solely dependent on the availability of internet access and the cost implication can be a barrier of adopting the system. The fourth reviewed work is closely related to this work since is offline but the issue of manually inputting student matriculation number before authentication gives rise to this offline and automatic $\mathrm{QR}$ code authentication.

\section{MATERIAL AND METHOD}

The proposed system was modeled using a Quick Response code and smartphone for dynamic authentication of embedded code. The quick response code is read by an imaging device, such as camera and authenticate by smartphone until the image can be appropriately interpreted. Data is then extracted from patterns present in both horizontal and vertical components of the image. The Figure below shows a sample of an unencrypted QR code that will be needed by the proposed system.

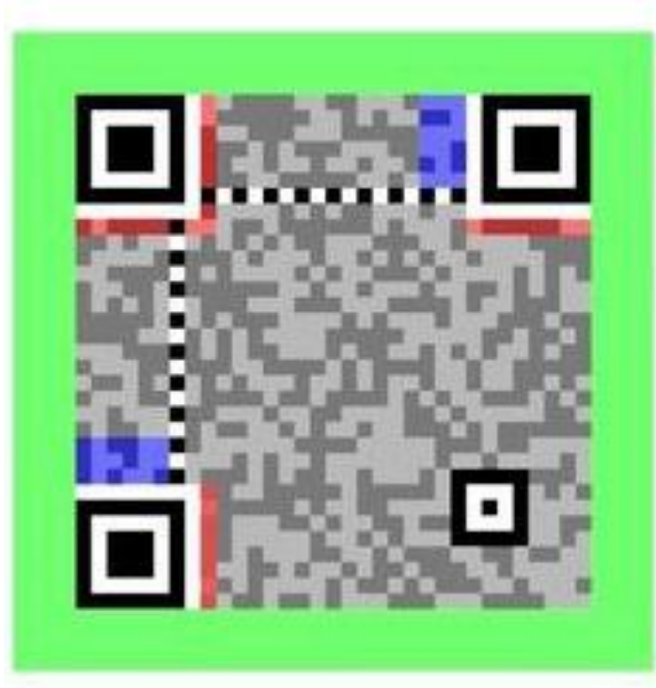

1. Version information

2. Format information

3. Data and error correction keys

4. Required patterns

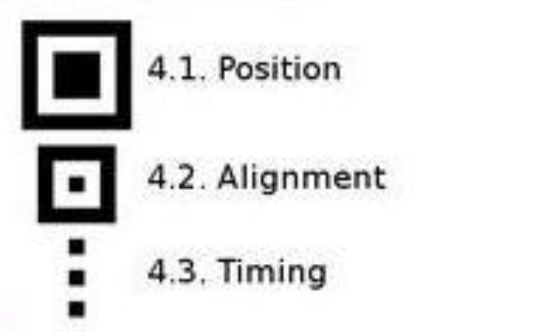

5. Quiet zone

Figure 1: The Structure of Quick Response Code (Ayeleso, et al., 2017) 


\section{The Proposed System Model}

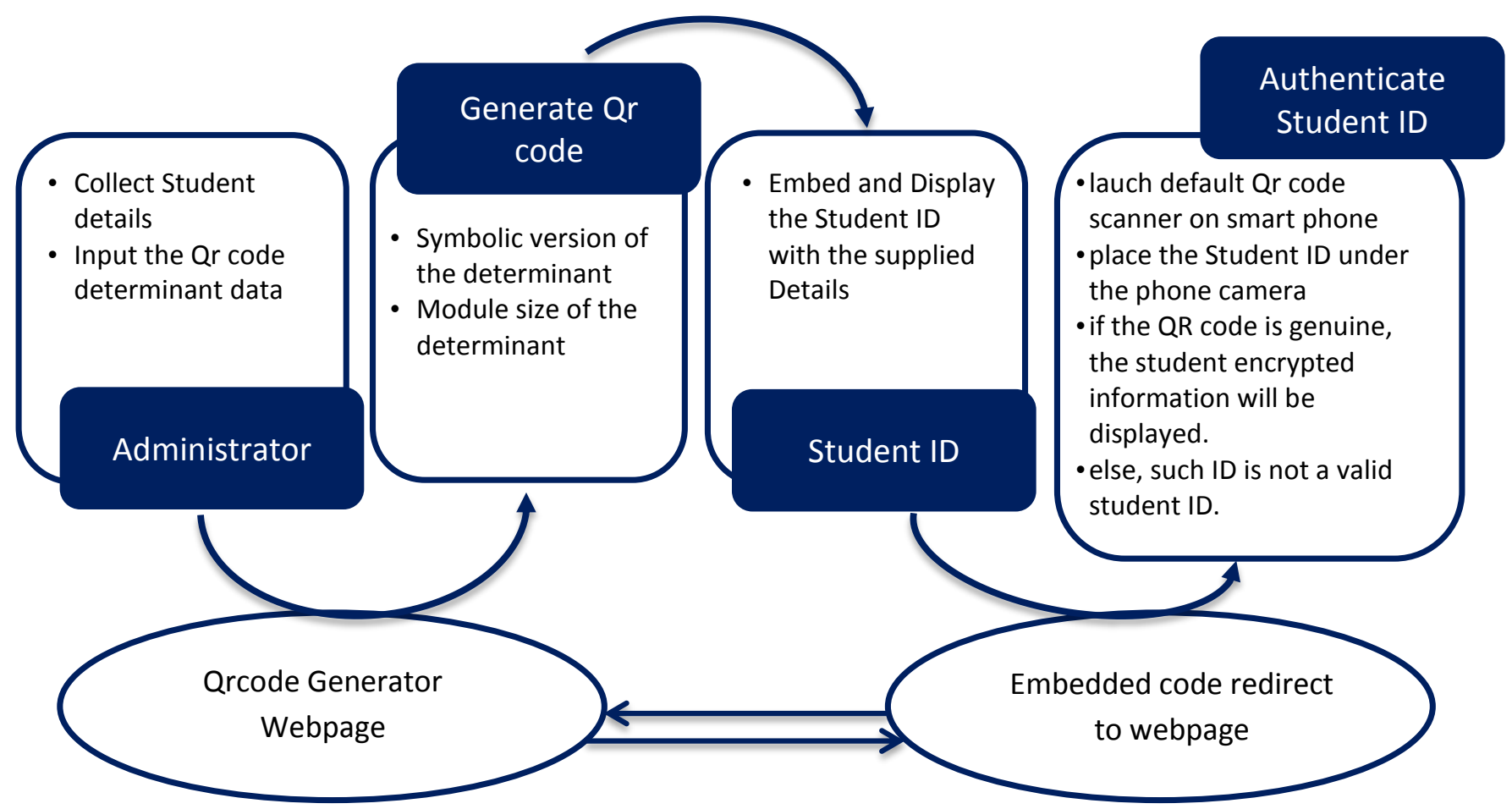

Figure 2: Model for the proposed system.

The proposed automatic authentication of student identity card using QR code and Smart Phone work as thus:

Collect the necessary student details during the registration processing.

Use the student unique value as an input process to generate and encrypt QR code.

Embed the generated QR code into the Student ID card.

Launch QR code Scanner on the smartphone

Place the Student ID card under the camera of the smartphone

The camera scans the embedded QR code and redirect to webpage before authenticates the Unique and encrypted value embedded on the Student ID.

\section{RESULT DISCUSSION}

This section serves the purpose of explaining all the program implementation and the entire process of program implementation. It covers areas such as input interface, output interface, menu interface, system testing, system maintenance, writing and testing of the computer program, routines that make up the new system to function very well and for it to achieve its objectives.

This is where the system administrator enters the username and password in other to gain access to the administrator control panel.

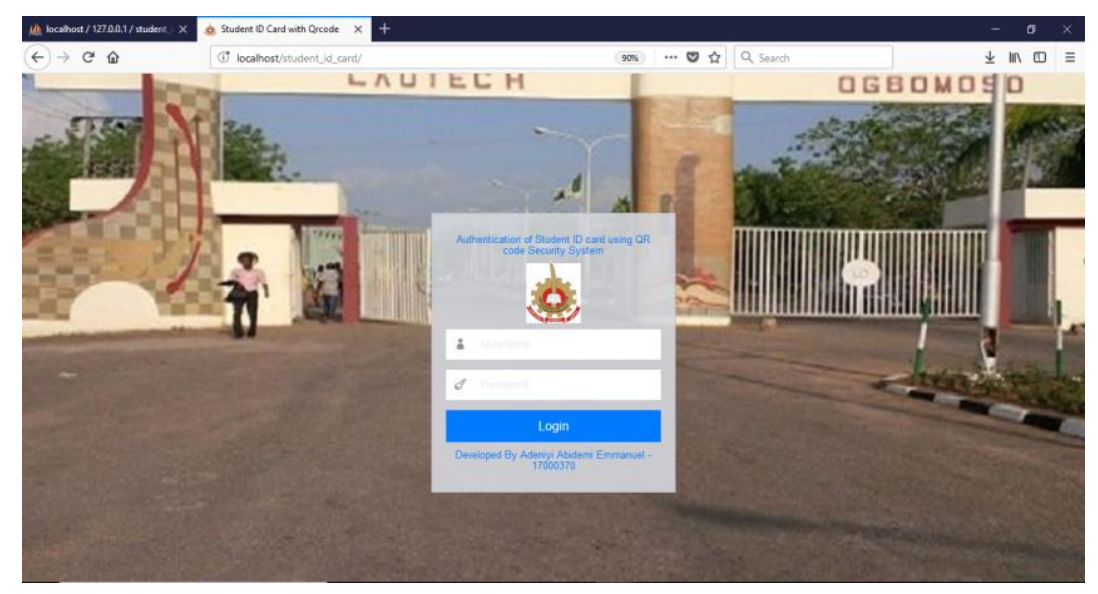

Figure 3: Home Page. 
International Journal of Engineering Research and Technology. ISSN 0974-3154, Volume 13, Number 1 (2020), pp. 48-53

(C) International Research Publication House. https://dx.doi.org/10.37624/IJERT/13.1.2020.48-53

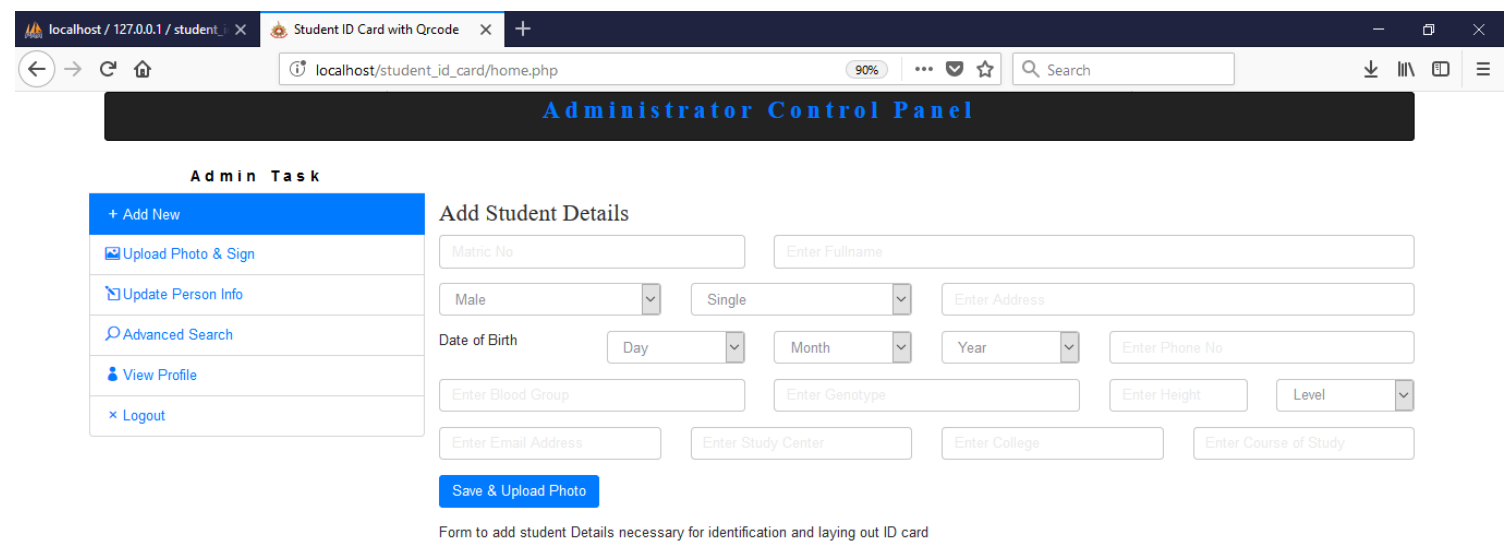

Figure 4: Add Student Details

This figure displays where basic information about the student will be entered before it proceeds to the upload of passport and signature.

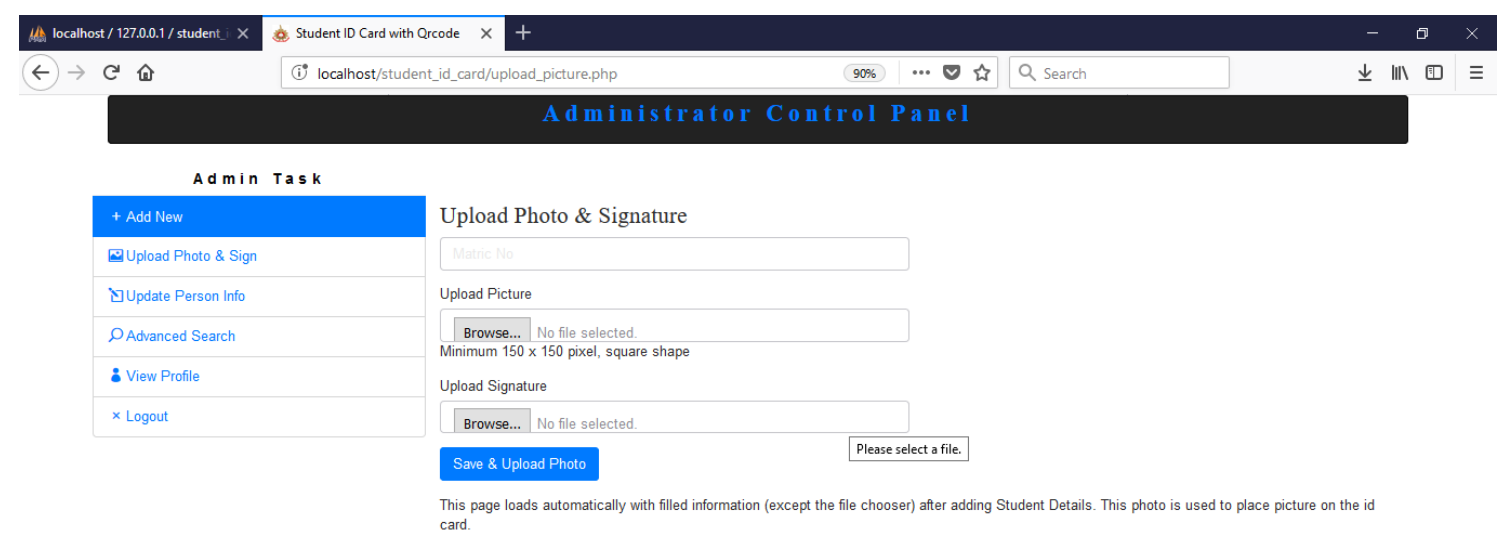

Figure 5: Passport and Signature Upload.

This figure displays where the student will upload his/her passport and signature before he/she proceeds to qrcode generation module.

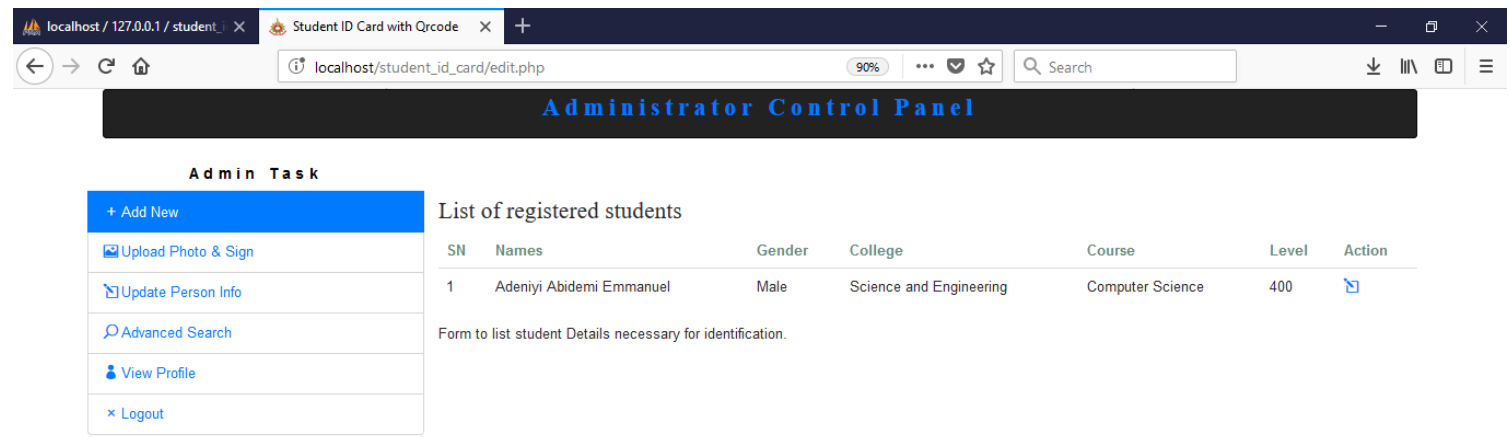

Figure 6: List of Registered Students

Figure 6 displays the list of all registered student that QR code

ID has been generated for. 
International Journal of Engineering Research and Technology. ISSN 0974-3154, Volume 13, Number 1 (2020), pp. 48-53

(C) International Research Publication House. https://dx.doi.org/10.37624/IJERT/13.1.2020.48-53

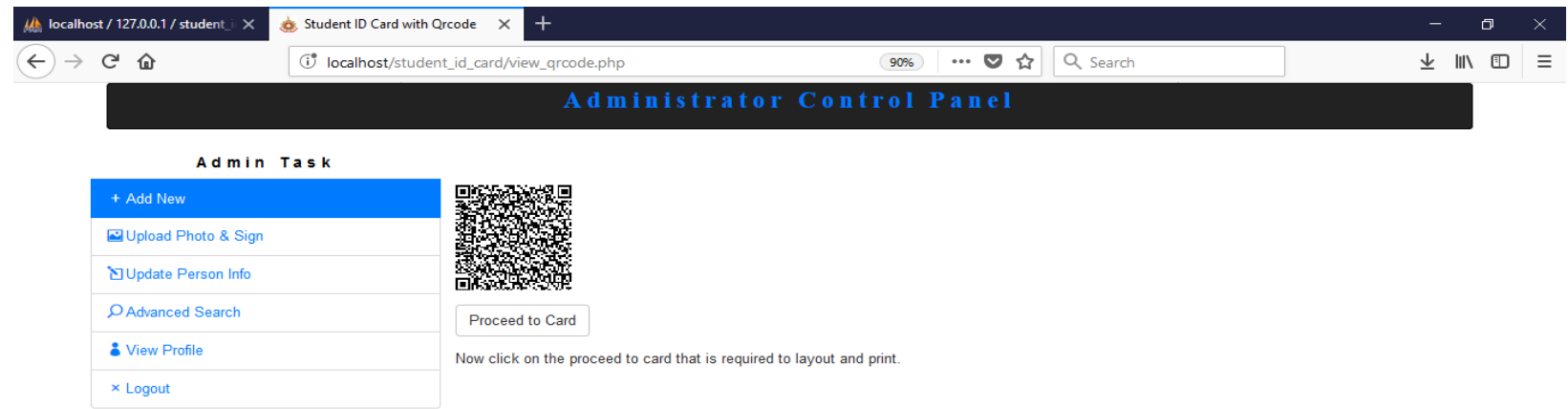

Figure 7: Qrcode Generated

This figure displays the generated quick response code which on click on the proceed to the card will embed the QR code into the identity card.

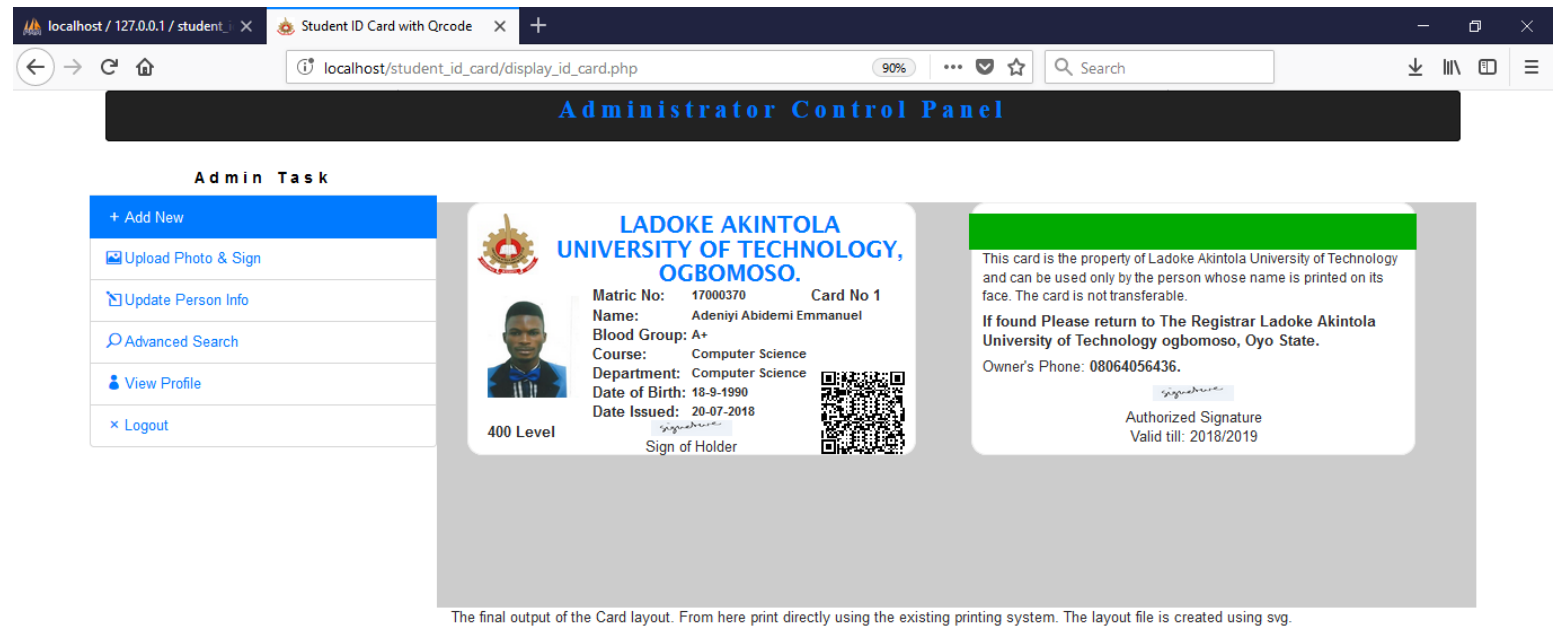

Figure 8: Generated Student Identity Card with Qrcode from student details.

This figure displays the student identity card from the student details and also embeds the generated quick response code from the student unique number.
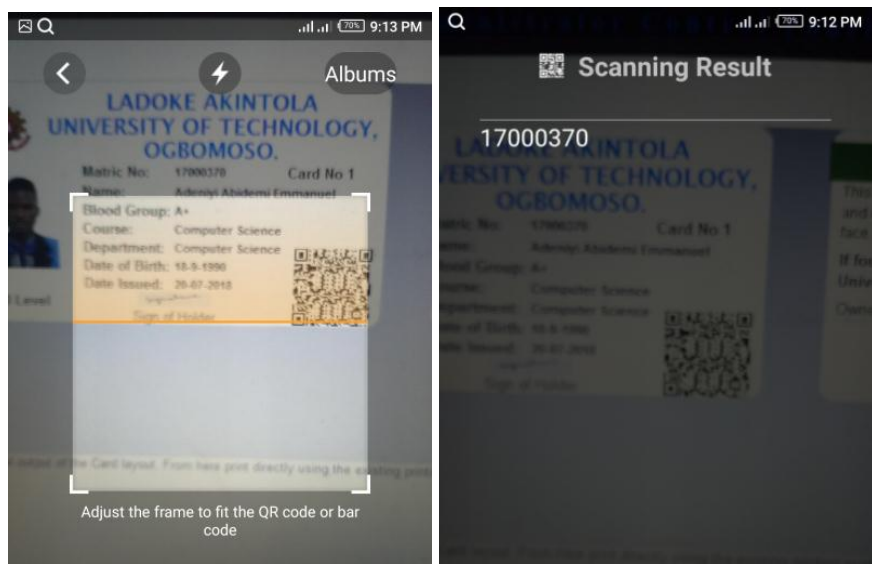

Figure 9: Student Identity Card with QR code authentication process.

Figure 9 displays how student identity card with the qrcode undergoes authentication process with the smartphone. The first image is the process of the authentication while the second image is the result of the QR code scanning. 
International Journal of Engineering Research and Technology. ISSN 0974-3154, Volume 13, Number 1 (2020), pp. 48-53

(C) International Research Publication House. https://dx.doi.org/10.37624/IJERT/13.1.2020.48-53

\section{Performance Goal}

Different scanners were used to carry out the experiment and it was discovered that there were no significant differences in the authentication processing using the different scanner but the time taken by each scanner makes the smartphone looks preferable than other since it take little time to verify the embedded code. That is, the type of tool used for authentication will determine how fast the process will take and it was discovered that subsequent scanning of this code of all devices takes little time compare to first scanning process.

Table 1: Experimental Results

\begin{tabular}{|l|l|}
\hline Scanner Used & Time in Seconds \\
\hline Android Phone & 3.22 \\
\hline Windows Phone & 4.50 \\
\hline Other Scanner & 4.00 average \\
\hline Samsung Tab & 3.34 \\
\hline
\end{tabular}

\section{CONCLUSION}

From the output of the discussion of result and experiment performed, it can be concluded that any smartphone with quick response code reader embedded will be able to capture the exact encrypted data faster during the authentication process. This aim of this research work was achieved. As the embedded code on the student identity card was able to be decrypted automatically with the aid of the smartphone quick response reader without the need for any manual input of previous data during the verification stage. In the future, this application can be applied to various activities that require verification and authentication. It can also be used in a supermarket for stock price list instead of the barcode.

\section{REFERENCES}

[1] Singhal, A., \& Pavithr, R. (2015). Degree Certificate Authentication using QR Code and Smartphone. International Journal of Computer Applications, 120(16), $38-43$.

[2] Cho, D., \& Bae, M. (2014). A Study on Development of OTIP System using QR Code based on Smartphone. International Journal of Multimedia and Ubiquitous Engineering, 9(10), 261-270. https://doi.org/http://dx.doi.org/10.14257/ijmue. 2014.9.10.25

[3] Ayeleso, et al., (2017). Identity card Authentication System Using QR code and Smartphone. International Journal of Science, Engineering and Environmental Technology. Vol. 2(9): 61-68. ISSN 0796-9650.

[4] Kumar, B. D., \& Kareemulla, S. (2017). Smart Mobile Attendance System for Employees Using QR Scanner. Asian Journal of Applied Science and Technology (AJAST), 1(5), 35-39.

[5] Kumar, B., Sharma, N., \& Yadav, N. (2014). Educational Application of QR (Quick Response) Code. International Journal of Advanced Research in Computer Science \& Technology (IJARCST 2014), 2(3), 373-375.

[6] Masalha, F., \& Hirzallah, N. (2014). A Students Attendance System Using QR Code. International Journal of Advanced Computer Science and Applications (IJACSA), 5(3), 75-79.

[7] Richard Dybowski. 2012. The Class Lectures. IT project. September-October. London: School of technology and Management.

[8] Bajpai, M, and Agrawal, A. (2013). Integration of 2D Barcode in Identity Cards: A New Approach. International Journal of Engineering and Computer Science. Vol. 2(4): 1225-1233. 